\title{
On the approach of a series to its Cesàro limit
}

\author{
By J. M. HysLop.
}

(Received 22nd November, 1937. Read 3rd December, 1937.)

(Received in revised form 4 th April, 1938.)

1.1. The object of this paper is to investigate some properties of series which satisfy conditions of the form

$$
S_{n}^{(p)}=O\left(n^{p-\rho}\right)
$$

or

$$
S_{n}^{(p)}=o\left(n^{p-\rho}\right),
$$

where $0<\rho \leqq p . \quad S_{n}^{(p)}$ denotes, as usual, the $n$-th Cesàro sum of order $p$ for the series $\Sigma a_{n}$ and $A_{n}^{(p)}$ the binomial coefficient $\left(\begin{array}{c}p+n \\ n\end{array}\right)$. It is convenient to state here some properties of $S_{n}^{(p)}$ and $A_{n}^{(p)}$ to which we must constantly refer in the sequel.

Lemma 1. The functions $S_{n}^{(p)}$ and $A_{n}^{(p)}$ possess the following properties:

$$
\begin{aligned}
& \sum_{\nu=0}^{n} A_{\nu}^{(p)} A_{n-\nu}^{(q)}=A_{n}^{(p+q+1)} \\
& \sum_{\nu=0}^{n} S_{\nu}^{(p)} A_{n-\nu}^{(q)}=S_{n}^{(p+q+1)} \\
& A_{n}^{(p)}-\frac{n^{p}}{\Gamma(p+1)}
\end{aligned}
$$

where $p$ is not a negative integer. If $p$ is a negative integer $A_{n}^{(p)}=0$ when $n \geqq-p$.

These results are all well known.

The relations (1.11) and (1.12) may be regarded, from our point of view, as simplified forms of the more extended relations

$$
\begin{aligned}
& S_{n}^{(p)}=s A_{n}^{(p)}+O\left(n^{p-\rho}\right), \\
& S_{n}^{(p)}=s A_{n}^{(p)}+\lambda A_{n}^{(p-\rho)}+o\left(n^{p-\rho}\right),
\end{aligned}
$$

where $0<\rho \leqq p$. More precisely we have the following lemma. 
LEMMA 2. If the series $\Sigma a_{n}$ satisfies (1.16) the series $\Sigma u_{n}$, where

$$
u_{n}=a_{n}-s A_{n}^{(-1)}
$$

satisfies (1.11). If $\Sigma a_{n}$ satisfies (1.17) the series $\Sigma v_{n}$, where

$$
v_{n}=a_{n}-s A_{n}^{(-1)}-\lambda A_{n}^{(-\rho-1)},
$$

satisfies (1.12).

Let $T_{n}^{(p)}$ be the $n$-th Cesàro sum of order $p$ for the series $\Sigma v_{n}$. Then, by (1.13) and (1.14),

$$
\begin{aligned}
T_{n}^{(p)} & =\sum_{\nu=0}^{n} A_{n-\nu}^{(p)} a_{\nu}-s \sum_{\nu=0}^{n} A_{n-\nu}^{(p)} A_{\nu}^{(-1)}-\lambda \sum_{\nu=0}^{n} A_{n-\nu}^{(p)} A_{\nu}^{(-p-1)} \\
& =S_{n}^{(p)}-s A_{n}^{(p)}-\lambda A_{n}^{(p-\rho)} \\
& =o\left(n^{p-\rho}\right) .
\end{aligned}
$$

The proof of the first result is obvious.

1.2. It will be observed that series which satisfy (1.16) or (1.17) are "more than" summable $(C, p)$. Generally speaking, the theorems of this paper may be regarded as analogues, for series which are " more than" summable $(C, p)$ of certain known" theorems for series which are "less than" summable $(C, p)$. It is convenient for purposes of reference to state these known theorems here.

Theorem 1. If $S_{n}^{(p)}=O\left(n^{p+\beta}\right), \beta>0$, then the series $\sum n^{-\gamma} a_{n}$ is summable $(C, p)$ for any $\gamma>\beta$.

Theorem 2. If $S_{n}^{(p)}=o\left(n^{p+\beta}\right), \beta>0$, then the series $\Sigma n^{-\beta} a_{n}$ is either summable $(C, p)$ or not summable by any Cesàro means.

Theorem 3. If the series $\Sigma n^{-\beta} a_{n}$ is summable $(C, p)$ and $\beta>0$ then $S_{n}^{(p)}=o\left(n^{p+\beta}\right)$.

In each case the theorem is proved on the supposition that $p$ is a positive integer. Whenever necessary in the interests of simplicity I shall impose the same restriction on $p$. All the results are probably true when $p$ is merely restricted to be positive, but their proofs, in the general case, would, I imagine, follow broadly the lines of Andersen's proof ${ }^{2}$ of the Bohr-Hardy Theorem, and be quite as long and difficult.

${ }^{1}$ Hardy and Littlewood, 7, 432-435. Dr L. S. Bosanquet kindly pointed out this connection to me, and his suggestion has enabled me to make this paper more comprehensive.

${ }^{2}$ Bohr, 2, 61. Hardy, 5. Andersen, 1, 47. 
It is clear that the most interesting cases of (1.16) or (1.17) occur when $0<\rho \leqq 1$. Indeed, if $\rho>1$ these conditions are very artificial and it would be difficult to find series to satisfy them, although it is easy enough, as we shall see, to find series satisfying the more extended conditions

$$
\begin{aligned}
& S_{n}^{(p)}=s A_{n}^{(p)}+\sum_{\sigma=1}^{r} \lambda_{\sigma} A_{n}^{p-\sigma}+O\left(n^{p-\rho}\right), \\
& S_{n}^{(p)}=s A_{n}^{(p)}+\sum_{\sigma=1}^{r} \lambda_{\sigma} A_{n}^{(p-\sigma)}+\lambda A_{n}^{(p-\rho)}+o\left(n^{p-\rho}\right),
\end{aligned}
$$

where $r=[\rho] \leqq \rho$, and $0<\rho \leqq p$.

The theorems of this paper may be modified to apply to series satisfying (1.21) or (1.22), but the presence of the terms involving $\lambda_{1}, \lambda_{2}, \ldots, \lambda_{r}$ involves a considerable amount of tedious algebraical work in their proofs. I have therefore considered it advisable to confine myself to the case $0<\rho \leqq 1$.

It will be shown that Theorem 1 remains true when $\beta<0$, but is capable of generalisation, that Theorem 2 remains unaltered with $\beta<0$, and that certain modifications have to be made in the case of Theorem 3 .

The paper concludes with some remarks on Tauberian Theorems for series satisfying these special conditions.

2.1. I begin by proving two simple theorems for series which satisfy (1.16) or (1.17).

Theorem 4. If (1.17) holds the series $\Sigma a_{n}$ is summable $(C, p-\rho)$ to the sum s, while, if (1.16) holds $\Sigma a_{n}$ is bounded $(C, p-\rho)$.

Suppose that (1.17) holds. Then, by (1.13) and (1.14),

$$
\begin{aligned}
S_{n_{*}}^{(p-\rho)} & =\sum_{\nu=0}^{n} A_{n-\nu}^{(-\rho-1)} S_{\nu}^{(p)} \\
& =s \sum_{\nu=0}^{n} A_{\nu}^{(p)} A_{n-\nu}^{(-\rho-1)}+\lambda \sum_{\nu=0}^{n} A_{\nu}^{(p-\rho)} A_{n-\nu}^{(-\rho-1)}+o\left\{\sum_{\nu=0}^{n} \nu^{p-\rho}(n-\nu+1)^{-\rho-1}\right\} \\
& =s A_{n}^{(p-\rho)}+\lambda A_{n}^{(p-2 \rho)}+o\left(n^{p-\rho}\right) \\
& =s A_{n}^{(p-\rho)}+o\left(n^{p-\rho}\right) .
\end{aligned}
$$

The proof is similar when (1.16) holds.

When (1.16) is satisfied the series $\Sigma a_{n}$ is bounded $(C, p-\rho)$ and also summable $(C, p)$. It follows, by a theorem ${ }^{1}$ due to Andersen,

Andersen, 1, 56. 
that $\Sigma a_{n}$ is summable $(C, p-\rho+\delta)$ for every positive $\delta$. It also follows that the index of summability of series $\Sigma a_{n}$, which satisfy (1.16) or (1.17), is not greater than $p-\rho$.

2.2. Theorem 5. If (1.16) or (1.17) is satisfied for a given value of $p$ it is also satisfied when $p$ is replaced by $p+\delta$ for any positive $\delta$.

Suppose that (1.17) is satisfied. Then, by Lemma 1,

$$
\begin{aligned}
S_{n}^{(p+\delta)} & =\sum_{\nu=0}^{n} A_{n-\nu}^{(\delta-1)} S_{\nu}^{(p)} \\
& =s \sum_{\nu=0}^{n} A_{\nu}^{(p)} A_{n-\nu}^{(\delta-1)}+\lambda \sum_{\nu=0}^{n} A_{\nu}^{(p-\rho)} A_{n-\nu}^{(\delta-1)}+o\left\{\sum_{\nu=0}^{n} A_{\nu}^{(p-\rho)} A_{n-\nu}^{(\delta-1)}\right\} \\
& =s A_{n}^{(p+\delta)}+\lambda A_{n}^{(p+\delta-\rho)}+o\left(n^{p+\delta-\rho}\right) .
\end{aligned}
$$

A similar proof holds in the case of (1.16).

2.3. As an illustration of these theorems and of some theorems which will be proved later on, it is interesting to consider the simple series $1-1+1 \ldots$ For this series, easy calculations show that

$$
\begin{aligned}
S_{n}= & \frac{1}{2}\left\{1+(-1)^{n}\right\}, \\
S_{n}^{(1)}= & \frac{1}{2} A_{n}^{(1)}+\frac{1}{4}\left\{1+(-1)^{n}\right\}, \\
S_{n}^{(2)}= & \frac{1}{2} A_{n}^{(2)}+\frac{1}{4} A_{n}^{(1)}+\frac{1}{8}\left\{1+(-1)^{n}\right\}, \\
& \cdots \cdots \cdots \cdots \cdots \cdots \cdots \cdots \cdots \cdots+\frac{1}{2^{p}} A_{n}^{(1)}+\frac{1}{2^{p+1}}\left\{1+(-1)^{n}\right\} . \\
S_{n}^{(p)}= & \frac{1}{2} A_{n}^{(p)}+\frac{1}{2^{2}} A_{n}^{(p-1)} \ldots \ldots
\end{aligned}
$$

Moreover, if $0<\delta<1$, we see from (2.33) that

where

$$
\begin{aligned}
S_{n}^{(1+\delta)} & =\frac{1}{2} \sum_{\nu=0}^{n} A_{n-\nu}^{(\delta-2)} A_{\nu}^{(2)}+\frac{1}{4} \sum_{\nu=0}^{n} A_{n-\nu}^{(\delta-2)} A_{\nu}^{(1)}+\frac{1}{8} \sum_{\nu=0}^{n} A_{n-\nu}^{(\delta-2)}\left\{1+(-1)^{\nu}\right\} \\
& =\frac{1}{2} A_{n}^{(1+\delta)}+\frac{1}{4} A_{n}^{(\delta)}+E
\end{aligned}
$$

Thus

$$
|E|<A \sum_{\nu=0}^{n}(\nu+1)^{-\delta-2}<A .
$$

Similarly it may easily be shown that

$$
S_{n}^{(\delta)}=\frac{1}{2} A_{n}^{(\delta)}+O(1) .
$$

3.1. In this section we consider the effect of the conditions of $\S 1$ on the summability of the series $\Sigma n^{\rho} a_{n}$ and similar series. Throughout, we shall denote by $S_{n, \rho}^{(p)}$ the $n$-th Cesaro sum of order $p$ for the series $\Sigma n^{\rho} a_{n}$, and by $T_{n, \rho}^{(p)}$ the $n$-th Cesàro sum of order $p$ for either 
$\Sigma n^{\rho} u_{n}$ or $\Sigma n^{\rho} v_{n}$. First of all we state a well known and easily proved lemma which connects the Cesàro sums of the series $\Sigma n a_{n}$ and $\Sigma a_{n}$.

\subsection{Lemma 3. If $p$ is positive we have}

$$
S_{n, 1}^{(p)}=(p+n+1) S_{n}^{(p)}-(p+1) S_{n}^{(p+1)} .
$$

We now make some straightforward deductions from Lemma 3.

Theorem 6. If $1=\rho \leqq p$ and $\Sigma a_{n}$ satisfies (1.17) then the series $\Sigma n a_{n}$ is summable $(C, p)$ to the sum $-\lambda$.

From Lemma 3 and Theorem 5 we have

$$
\begin{aligned}
S_{n, 1}^{(p)}= & (p+n+1)\left\{s A_{n}^{(p)}+\lambda A_{n}^{(p-1)}+o\left(n^{p-1}\right)\right\} \\
& \quad-(p+1)\left\{s A_{n}^{(p+1)}+\lambda A_{n}^{(p)}+o\left(n^{p}\right)\right\} \\
= & \lambda\left\{(p+n+1) A_{n}^{(p-1)}-(p+1) A_{n}^{(p)}\right\}+o\left(n^{p}\right) \\
= & \lambda A_{n}^{(p)}\left\{(p+n+1) \frac{A_{n}^{(p-1)}}{A_{n}^{(p)}}-(p+1)\right\}+o\left(n^{p}\right) \\
= & -\lambda A_{n}^{(p)}\left\{(p+1)-\frac{p(p+n+1)}{p+n}\right\}+o\left(n^{p}\right) \\
= & -\lambda A_{n}^{(p)}+o\left(n^{p}\right) .
\end{aligned}
$$

A similar, though simpler, proof suffices to prove the following theorem.

THEOREM 7. If $1=\rho \leqq p$ and $\Sigma a_{n}$ satisfies (1.16), then the series $\Sigma n a_{n}$ is bounded $(C, p)$.

If $\Sigma a_{n}$ is the particular series $1-1+1-\ldots$ we see from (2.32) and Theorem 7 that $\Sigma n a_{n}$ is bounded $(C, 1)$, while, from (2.33) and Theorem $6, \Sigma n a_{n}$ is summable $(C, 2)$ to the sum $-\frac{1}{4}$. Using Andersen's Theorem ${ }^{1}$ we at once obtain the familiar result that the series $1-2+3-4 \ldots$ is summable $(C, 1+\delta)$, to the sum $\frac{1}{4}$, for every positive $\delta$.

3.3. We now prove two theorems similar to Theorems 6 and 7 for the case $0<\rho<1$. The proof of Theorem 8 may easily be constructed from that of Theorem 9 , so that it is only necessary to prove the latter. In the proof of this and some succeeding theorems

${ }^{1}$ Andersen, 1, 56. 
we employ a technique which was introduced ${ }^{1}$ and developed by Andersen.

Lemma 4. If $s$ is any positive integer and $r$ is any real number ${ }^{2}$, then

where

$$
\sum_{p=0}^{\delta} \Delta_{\nu+p}^{\delta+1} \epsilon_{\nu} A_{n-\nu-p}^{(r-1)}=\sum_{q=0}^{\delta}\left(\begin{array}{c}
s+1 \\
q
\end{array}\right) \Delta^{q} \epsilon_{\nu} A_{n-\nu-q}^{(r+q-8-1)},
$$

$$
\begin{aligned}
& \Delta^{s+1} \epsilon_{\nu}=\epsilon_{\nu}-\left(\begin{array}{c}
s+1 \\
1
\end{array}\right) \epsilon_{\nu+1}+\left(\begin{array}{c}
s+1 \\
2
\end{array}\right) \epsilon_{\nu+2}+\ldots+(-1)^{s+1} \epsilon_{\nu+s+1}, \\
& \Delta_{\nu+p}^{s+1} \epsilon_{\nu}=\epsilon_{\nu}-\left(\begin{array}{c}
s+1 \\
1
\end{array}\right) \epsilon_{\nu+1}+\ldots+(-1)^{p}\left(\begin{array}{c}
s+1 \\
p
\end{array}\right) \epsilon_{\nu+p} .
\end{aligned}
$$

LEMMA 5. If $r$ is any real number, and $p$ is a positive integer, we have

$$
\sum_{\nu=\mu}^{n} A_{n-\nu}^{(r)} A_{\nu-\mu}^{(-p-2)} \epsilon_{\nu}=\sum_{q=0}^{p+1}\left(\begin{array}{c}
p+1 \\
q
\end{array}\right) A_{n-\mu-q}^{(r+q-p-1)} \Delta^{q} \epsilon_{\mu} .
$$

We have

$$
\begin{aligned}
\sum_{\nu=\mu}^{n} A_{n-\nu}^{(r)} A_{\nu-\mu}^{(-p-2)} \epsilon_{\nu} & =\sum_{\sigma=0}^{n-\mu} A_{n-\mu-\sigma}^{(r)} A_{\sigma}^{(-p-2)} \epsilon_{\mu+\sigma} \\
& =\sum_{\sigma=0}^{n-\mu} A_{\sigma}^{(-p-2)} \epsilon_{\mu+\sigma} \sum_{\tau=0}^{n-\mu-\sigma} A_{\tau}^{(r-1)} \\
& =\sum_{\tau=0}^{n-\mu} A_{\tau}^{(r-1)} \Delta_{n-\tau}^{p+1} \epsilon_{\mu} \\
& =\sum_{\tau=0}^{n-\mu} A_{n-\mu-\tau}^{(r-1)} \Delta_{\mu+\tau}^{p+1} \epsilon_{\mu} \\
& =\sum_{\tau=0}^{p} A_{n-\mu-\tau}^{(r-1)} \Delta_{\mu+\tau}^{p+1} \epsilon_{\mu}+\Delta^{p+1} \epsilon_{\mu} \sum_{\tau=p+1}^{n-\mu} A_{n-\mu-\tau}^{(r-1)},
\end{aligned}
$$

and, by Lemma 4, this is equal to

$$
\begin{aligned}
& \sum_{q=0}^{p}\left(\begin{array}{c}
p+1 \\
q
\end{array}\right) A_{n-\mu-q}^{(r+q-p-1)} \Delta \epsilon_{\mu}+A_{n-\mu-p-1}^{(r)} \Delta^{p+1} \epsilon_{\mu} \\
= & \sum_{q=0}^{p+1}\left(\begin{array}{c}
p+1 \\
q
\end{array}\right) A_{n-\mu-q}^{(r+q-p-1)} \Delta^{q} \epsilon_{\mu} .
\end{aligned}
$$

The essence of this result is contained in Andersen's dissertation, but it is not quite stated in the form (3.32) which is most convenient for our purpose. In these circumstances I considered it advisable to insert the above proof.

1 Andersen, 1, 47.

2 Andersen, 1, 42. 
ThEOREM 8. If $p$ is a positive integer and if $\Sigma a_{n}$ satisfies (1.17), then, for $0<\delta<\rho \leqq 1 \leqq p$, the series $\sum n^{\rho-\delta} a_{n}$ is summable $(C, p-\delta)$ to the sum of the convergent series

$$
\lambda \sum_{\nu=0}^{\infty} A_{\nu}^{(-\rho-1)} \nu^{\rho-\delta}+\sum_{\nu=0}^{\infty} \eta_{\nu} \Delta^{p+1} \nu^{\rho-\delta},
$$

where $\eta_{n}$ denotes the error term in (1.17).

TheOREM 9. If $p$ is a positive integer and if $\Sigma a_{n}$ satisfies (1.16) then, for $0<\delta<\rho \leqq 1 \leqq p$, the series $\Sigma n^{\rho-\delta} a_{n}$ is bounded $(C, p-\delta)$, and summable $(C, p-\delta+\epsilon)$, for any positive $\epsilon$, to the sum of the convergent series

$$
\sum_{\nu=0}^{\infty} \beta_{\nu} \Delta^{p+1} \nu^{\rho-\delta}
$$

where $\beta_{n}$ denotes the error term in (1.16).

It should be observed that these theorems are also true when $\delta=\rho$ and have already been proved as parts of Theorem 4. In this case, of course, the sum of the resulting series, namely $\Sigma a_{n}$, is $s$, so that (3.33) and (3.34) do not apply.

For the series $\Sigma u_{n}$, defined as in (1.18), we have

and

$$
T_{n}^{(p)}=\beta_{n}=O\left(n^{p-\rho}\right)
$$

$$
\begin{aligned}
T_{n, \rho-\delta}^{(p-\delta+\epsilon)} & =\sum_{\nu=0}^{n} A_{n-\nu}^{(p-\delta+\epsilon)} \nu^{\rho-\delta} a_{\nu} \\
& =\sum_{\nu=0}^{n} A_{n-\nu}^{(p-\delta+\epsilon)} \nu^{\rho-\delta} \sum_{\mu=0}^{\nu} A_{\nu-\mu}^{(-p-2)} T_{\mu}^{(p)} \\
& =\sum_{\mu=0}^{n} T_{\mu}^{(p)} \sum_{\nu=\mu}^{n} \nu^{\rho-\delta} A_{n-\nu}^{(p-\delta+\epsilon)} A_{\nu-\mu}^{(-p-2)} \\
& =E_{1}+E_{2},
\end{aligned}
$$

where

$$
E_{1}=\sum_{\mu=n-p}^{n} T_{\mu}^{(p)} \sum_{\nu=\mu}^{n} A_{n-\nu}^{(p-\delta+\epsilon)} \nu^{\rho-\delta} A_{\nu-\mu}^{(-p-2)}=O\left(n^{p-\delta}\right)
$$

and

$$
E_{2}=\sum_{\mu=0}^{n-p-1} \beta_{\mu} \sum_{\nu=\mu}^{n} A_{n-\nu}^{(p-\delta+\varepsilon)} \nu^{\rho-\delta} A_{\nu-\mu}^{(-p-2)}
$$

By Lemma 5, we may write

$$
E_{2}=\sum_{q=0}^{p+1}\left(\begin{array}{c}
p+1 \\
q
\end{array}\right) F_{q}
$$


where

Since

$$
F_{q}=\sum_{\mu=0}^{n-p-1} \beta_{\mu} A_{n-\mu-q}^{(q+e-\delta-1)} \Delta^{q} \mu^{p-\delta}
$$

we have

$$
\Delta^{q} \mu^{p-\delta}=O\left(\mu^{p-\delta-q}\right)
$$

$$
\begin{aligned}
F_{0} & =O\left\{\sum_{\mu=0}^{n-p-1} \mu^{p-\delta}(n-\mu)^{\epsilon-\delta-1}\right\} \\
& =O\left\{n^{p-\delta} \sum_{\mu=0}^{\infty}(\mu+1)^{\epsilon-\delta-1}\right\} \\
& =O\left(n^{p-\delta}\right),
\end{aligned}
$$

if we suppose, as we may without loss of generality, that $0<\epsilon<\delta$.

Again, if $1 \leqq q \leqq p$,

since $0<\epsilon<\delta$.

$$
\begin{aligned}
F_{q} & =O\left\{\sum_{\mu=0}^{n-q} A_{\mu}^{(p-\delta-q)} A_{n-q-\mu}^{(q+\epsilon-\delta-1)}\right\}+O\left(n^{p-\delta}\right) \\
& =O\left\{A_{n-q}^{(p+\varepsilon-2 \delta)}\right\}+O\left(n^{p-\delta}\right) \\
& =O\left(n^{p-\delta}\right)
\end{aligned}
$$

Finally

$$
\frac{F_{p+1}}{A_{n-p-1}^{(p-\delta+\epsilon)}}=\frac{1}{A_{n-p-1}^{(p-\delta+\epsilon)}} \sum_{\mu=0}^{n-p-1} \beta_{\mu} \Delta^{p+1} \mu^{\rho-\delta} A_{n-p-1-\mu}^{(p-\delta+\epsilon)},
$$

which, by the consistency theorem for Cesàro summability, tends to the sum of the convergent series

$$
\sum_{\nu=0}^{\infty} \beta_{\nu} \Delta^{p+1} \nu^{\rho-\delta}
$$

The second part of the theorem now follows almost at once.

It has in fact been shown that

$$
S_{n, p-\delta}^{(p-\delta+\epsilon)}=A_{n}^{(p-\delta+\epsilon)} \sum_{\nu=0}^{\infty} \beta_{\nu} \Delta^{p+1} \nu^{\rho-\delta}+O\left(n^{p-\delta}\right)
$$

and the first part of the theorem follows from this and Theorem 4.

It is interesting at this point to compare Theorem 9 with the case of Theorem 1 when $\beta<0$. Translating Theorem 1 with $-1<\beta<0$ into the notation of the present paper it would become:-

If $\Sigma a_{n}$ satisfies (1.11) then, for $0<\delta \leqq \rho<1 \leqq p$, where $p$ is $a$ positive integer, the series $\Sigma n^{\rho-\delta} a_{n}$ is summable $(C, p)$.

It will be observed that Theorem 9 is more general than this result. 
4.1. It is easy to see that the hypotheses of Theorems 8 and 9 do not necessarily imply that $\Sigma n^{\rho} a_{n}$ is summable or bounded $(C, p)$. If, for example,

we have

$$
a_{n}=A_{n}^{(-3)} \text {, }
$$

$$
S_{n}^{(1)}=A_{n}^{\left(\frac{1}{n}\right)} \text {, }
$$

and the series $\Sigma n^{\frac{1}{2}} a_{n}$, being properly divergent, is neitner summable nor bounded $(C, 1)$. The example indeed shows that, in general, it is not possible to assert that if $\Sigma a_{n}$ satisfies (1.16), or (1.17), the series $\sum n^{\rho} a_{n}$ will be bounded, or summable, by some Cesàro means of sufficiently high order. In this respect the cases $0<\rho<1$ and $\rho=1$ of (1.16) and (1.17) present a marked contrast.

4.2. In view of these remarks the following theorems are of interest.

Theorem 10. If $p$ is a positive integer, $0<\rho<1 \leqq p$, and $\Sigma a_{n}$ satisfies (1.16), then the series $\Sigma n^{\rho} a_{n}$ is either bounded $(C, p)$ or not bounded by any Cesìro means.

Theorem 11. If $p$ is a positive integer, $0<\rho<1 \leqq p$, and $\Sigma a_{n}$ satisfies (1.17), with $\lambda=0$, then the series $\Sigma n^{\rho} a_{n}$ is either summable $(C, p)$ or not summable by any of Cesàro's means.

It is well known and, in any case, follows readily from Lemma 3 and the consistency theorem for Cesàro summability, that necessary and sufficient conditions for a series $\Sigma a_{n}$ to be summable $(C, p)$ are that $\Sigma a_{n}$ should be summable by some Cesàro method and that the sequence $n a_{n}$ should be summable $(C, p+1)$ to zero. A similar result holds for series to be bounded $(C, p)$.

It should be observed that, if we take the series $\Sigma v_{n}$ instead of $\Sigma a_{n}$, Theorem 11 corresponds exactly to the case of Theorem 2 when $-\mathrm{I}<\beta<0$. Hardy and Littlewood's proof extends to this case without alteration but, for the sake of completeness, I give a brief proof on the lines of the proof of Theorem 9. Theorem 10 may, of course, be proved by the same method.

It is sufficient to show that

$$
S_{n, p+1}^{(p)}=o\left(n^{p+1}\right) .
$$

Taking as our hypothesis

$$
S_{n}^{(p)}=s A_{n}^{(p)}+\eta_{n}, \eta_{n}=o\left(n^{p-\rho}\right),
$$


we have

$$
\begin{aligned}
S_{n, \rho+1}^{(p)} & =\sum_{\nu=0}^{n} A_{n-\nu}^{(p)} \nu^{\rho+1} \sum_{\mu=0}^{\nu} A_{\nu-\mu}^{(-p-2)} S_{\mu}^{(p)} \\
& =\sum_{\mu=0}^{n} \eta_{\mu} \sum_{\nu=\mu}^{n} A_{n-\nu}^{(p)} A_{\nu-\mu}^{(-p-2)} \nu^{\rho+1} \\
& =0\left(n^{p+1}\right)+\sum_{\mu=0}^{n-p-1} \eta_{\mu} \sum_{\nu=\mu}^{n} A_{n-\nu}^{(p)} A_{\nu-\mu}^{(-p-2)} \nu^{\rho+1}
\end{aligned}
$$

By Lemma 5, the second term may be written in the form

where

$$
\sum_{q=0}^{p+1}\left(\begin{array}{c}
p+1 \\
q
\end{array}\right) F_{q},
$$

$$
F_{q}=\sum_{\mu=0}^{n-p-1} \eta_{\mu} A_{n-\mu-q}^{(q-1)} \Delta^{q} \mu^{\rho+1}, \quad q=0,1,2, \ldots, p+1
$$

Clearly $F_{0}=0$ and, for $1 \leqq q \leqq p+1$,

$$
F_{q}=o\left\{\sum_{\mu=0}^{n-p-1} A_{n-\mu-q}^{(q-1)} \mu^{p+1-q}\right\}=o\left(n^{p+1}\right) .
$$

When $\Sigma a_{n}$ satisfies (1.17) and $\lambda \neq 0$ we have the following more precise theorem.

Theorem 12. If $p$ is a positive integer, $0<\rho<1 \leqq p$, and $\Sigma a_{n}$ satisfies (1.17), with $\lambda \neq 0$, then the series $\Sigma n^{\rho} a_{n}$ is not summable by Cesiro means of any order.

If $k$ is any number $\geqq p$ and $\eta_{n}$, as usual, denotes the error term in (1.17), we have, by arguments similar to those used in the proofs of Theorems 9 and 11 ,

$$
\begin{aligned}
S_{n, \rho}^{(k)} & =\lambda \sum_{\nu=0}^{n} A_{n-\nu}^{(k)} A_{\nu}^{(-\rho-1)} \nu^{\rho}+\sum_{\mu=0}^{n-p-1} \eta_{\mu} A_{n-p-1-\mu}^{(k)} \Delta^{p+1} \mu^{\rho}+o\left(n^{k}\right) \\
& =\lambda \sum_{\nu=0}^{n} A_{n-\nu}^{(k)} A_{\nu}^{(-\rho-1)} \nu^{\rho}+\sum_{\nu=p+1}^{n} \epsilon_{\nu-p-1}(\nu-p-1)^{p-\rho} A_{n-\nu}^{(k)} \Delta^{p+1}(\nu-p-1)^{\rho}+o\left(n^{k}\right),
\end{aligned}
$$

where $\epsilon_{n} \rightarrow 0$ as $n \rightarrow \infty$. Now

$$
\begin{gathered}
A_{\nu}^{(-\rho-1)}=A \nu^{-\rho-1}+O\left(\nu^{-\rho-2}\right), \\
(\nu-p-1)^{p-\rho} \Delta^{p+1}(\nu-p-1)^{\rho}=B \nu^{-1}+O\left(\nu^{-2}\right),
\end{gathered}
$$

where $A$ and $B$ are non-zero constants. Given $\epsilon\left(<\frac{A \lambda}{B} \mid\right)$, we can 
determine $N(>p+1)$, such that $\left|\epsilon_{\nu-p-1}\right|<\epsilon$ for $v \geqq N$. Thus, since $A_{n}^{(-p-1)}<0$ for $n \geqq 1$, we have

$$
\begin{aligned}
\left|S_{n, \rho}^{(k)}\right| & \geqq\left|A \lambda \sum_{\nu=N}^{n} A_{n \rightarrow \nu}^{(k)} v^{-1}\right|-\left|\sum_{\nu=N}^{n} B \epsilon_{\nu-p-1} A_{n-\nu}^{(k)} \nu^{-1}\right|-O\left(n^{k}\right) \\
& \geqq\{|A \lambda|-|B| \epsilon\} \sum_{\nu=N}^{n} A_{n-\nu}^{(k)} v^{-1}-O\left(n^{k}\right) .
\end{aligned}
$$

Since the series $\Sigma v^{-1}$ is properly divergent, the result follows.

4.3 We have seen from Theorem 9 that, if $\Sigma a_{n}$ satisfies (1.16) and $0<\delta \leqq \rho<1 \leqq p$, the series $\Sigma n^{\rho-\delta} a_{n}$ is summable $(C, p-\delta+\epsilon)$ for every positive $\epsilon$. Also, from Theorem 7 , we know that, if $\Sigma a_{n}$ satisfies (1.16) when $p \geqq \rho=1$, the series $\Sigma n a_{n}$ is bounded $(C, p)$. It might be plausible then to suppose that the hypothesis of Theorem 7 would imply the summability $(C, p+\epsilon)$ of $\Sigma n a_{n}$ for every positive $\epsilon$. Such a surmise however would be erroneous for it is possible to construct a series $\Sigma a_{n}$, which satisfies (1.16) in the particular case $p=\rho=1$, and for which the series $\Sigma n a_{n}$ is not summable $(C, 1+\epsilon)$ for any positive $\epsilon$.

Consider, for example, the series $\Sigma a_{n}$ for which

$$
S_{n}^{(1)}=\lambda_{n},
$$

where $\lambda_{n}$ is the bounded function ${ }^{1}$

$$
\begin{array}{rlrl}
\lambda_{n} & =1, & & 2^{2 v} \leqq n<2^{2 \nu+1} \\
& =0, & 2^{2 v+1} \leqq n<2^{2 \nu+2}
\end{array}
$$

As in the proof of Theorem 9, we have

$$
\begin{aligned}
S_{n, 1}^{(2)} & =\sum_{\mu=0}^{n} \lambda_{\mu} \sum_{\nu=\mu}^{n} \nu A_{n-r}^{(2)} A_{\nu-\mu}^{(-3)} \\
& =\sum_{\mu=0}^{n} \lambda_{\mu}(3 \mu-2 n) .
\end{aligned}
$$

It is not difficult to show that

$$
\left.\left.n^{-1} \sum_{\mu=0}^{n} \lambda_{\mu} \rightarrow \begin{array}{c}
\frac{1}{\vdots} \\
\frac{2}{\sharp}
\end{array}\right\}, \quad n^{-2} \sum_{\mu=0}^{n} \mu \lambda_{\mu} \rightarrow \begin{array}{c}
\frac{1}{10} \\
\frac{2}{\overline{5}}
\end{array}\right\},
$$

according as $n$ tends to infinity through the even or the odd powers of 2. It follows that

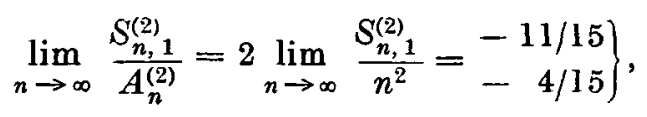

${ }^{1}$ The series $\Sigma\left(\lambda_{n}-\lambda_{n-1}\right)$ possesses many interesting properties. See Hardy, 4, 6 . 
according as $n$ tends to infinity through the even or the odd powers of 2. Thus the series $\Sigma n a_{n}$ is not summable $(C, 2)$ and a fortiori is not summable $(C, 1+\epsilon)$ for $0<\epsilon<1$. Moreover $\Sigma n a_{n}$ is not summable $(C, 1+\epsilon)$ for $\epsilon>1$, for, if it were, it would necessarily be summable $(C, 2)$ since, by Theorem 7 , it is bounded $(C, 1)$.

4.4. We may appropriately conclude this section by remarking that, if we apply Theorem 9 to the series $1-1+1 \ldots$, taking as hypothesis the relation (2.32), we obtain the following familiar result: For $0<\delta \leqq 1$, the series

$$
1^{1-\delta}-2^{1-\delta}+3^{1-\delta} \ldots
$$

is bounded $(C, 1-\delta)$ and summable $(C, 1-\delta+\epsilon)$ for every positive $\epsilon$.

5.1. We next consider the case of Theorem 3 when $-1 \leqq \beta<0$. The problem discussed is the converse of the type of result which is contained in Theorems 6, 7,8 and 9; that is, we assume as hypothesis the summability or boundedness $(C, p)$ of the series $\Sigma n^{\rho} a_{n}$ and the conclusion involves a property of $\Sigma a_{n}$. One well known result in this connection is that the summability $(C, p)$ of $\Sigma n^{\rho} a_{n}$ implies the summability $(C, p-\delta)$ of $\Sigma n^{\rho-\delta} a_{n}$, where $0<\delta \leqq \rho \leqq p$, and the results of this section may be regarded as generalisations of theorems of this kind for the particular case $0<\delta=\rho \leqq 1$.

5.2. First we establish a lemma which serves to simplify the proofs of the theorems which follow.

LEMMA 6. If $\gamma>0$ and

$$
\phi_{m}(\gamma, a)=\sum_{\mu=1}^{m}\left\{A_{m}^{(\gamma)}-A_{m-\mu}^{(\gamma)}\right\} \epsilon_{\mu} \mu^{-a-1}, \quad \epsilon_{\mu} \rightarrow 0
$$

then

$$
\begin{aligned}
& =o\left(m^{\gamma-a}\right) & , & 0<a<1, \\
\phi_{m}(\gamma, a) & =o\left(m^{\gamma-1} \log m\right), & & a=1, \\
& =O\left(m^{\gamma-1}\right) & , & a>1 .
\end{aligned}
$$

We have

$$
\begin{aligned}
\phi_{m}(\gamma, \alpha) & =\sum_{\mu=1}^{m} \epsilon_{\mu} \mu^{-a-1} \sum_{\tau=m+1-\mu}^{m} A_{\tau}^{(\gamma-1)} \\
& =\sum_{\tau=1}^{m} A_{\tau}^{(\gamma-1)} \sum_{\mu=m+1-\tau}^{m} \epsilon_{\mu} \mu^{-a-1} .
\end{aligned}
$$


Given $\epsilon$, there exists $N$ such that, for $\mu \geqq N,\left|\epsilon_{\mu}\right|<\epsilon$. Thus

$$
\begin{aligned}
\left|\phi_{m}(\gamma, a)\right| \leqq & \sum_{\tau=1}^{m+1-N} A_{\tau}^{(\gamma-1)} \sum_{\mu=m+1-\tau}^{m} \epsilon_{\mu} \mu^{-a-1} \mid+\sum_{\tau=m+2-N}^{m} A_{\tau}^{(\gamma-1)} \sum_{\mu=m+1-\tau}^{m} \epsilon_{\mu} \mu^{-a-1} \\
& <\epsilon \sum_{\tau=1}^{m+1-N} A_{\tau}^{(\gamma-1)} \sum_{\mu=m+1-\tau}^{\infty} \mu^{-a-1}+O\left(m^{\gamma-1}\right) .
\end{aligned}
$$

Hence, if $0<a<1$,

$$
\begin{aligned}
\phi_{m}(\gamma, \alpha) & =o\left\{\sum_{\tau=1}^{m+1} A_{\tau}^{(\gamma-1)} A_{m+1-\tau}^{(--a)}\right\}+O\left(m^{\gamma-1}\right) \\
& =o\left(m^{\gamma-\alpha}\right),
\end{aligned}
$$

while, if $a>1$,

$$
\begin{aligned}
\phi_{m}(\gamma, a) & =o\left\{\sum_{\tau=1}^{m} A_{\tau}^{(\gamma-1)}(m+1-\tau)^{-a}\right\}+O\left(m^{\gamma-1}\right) \\
& =o\left[\left\{\sum_{\tau=1}^{\frac{1}{m} m}+\sum_{\tau=\frac{1}{2} m}^{m}\right\} A_{\tau}^{(\gamma-1)}(m+1-\tau)^{-a}\right]+O\left(m^{\gamma-1}\right) \\
& =o\left\{m^{-a} \sum_{\tau=1}^{\frac{1}{2} m} A_{\tau}^{(\gamma-1)}\right\}+o\left\{m^{\gamma-1} \sum_{\tau=1}^{\infty} \tau^{-a}\right\}+O\left(m^{\gamma-1}\right) \\
& =O\left(m^{\gamma-1}\right) .
\end{aligned}
$$

Finally, if $a=1$,

$$
\begin{aligned}
\phi_{m}(\gamma, a) & =o\left\{m^{-1} \sum_{\tau=1}^{\frac{1}{2} m} A_{\tau}^{(\gamma-1)}\right\}+o\left\{m^{\gamma-1} \sum_{\tau=1}^{\frac{1}{2} m} \tau^{-1}\right\}+O\left(m^{\gamma-1}\right) \\
& =o\left(m^{\gamma-1}\right)+o\left(m^{\gamma-1} \log m\right)+O\left(m^{\gamma-1}\right) \\
& =o\left(m^{\gamma-1} \log m\right) .
\end{aligned}
$$

5.3. THEOREM 13. If $p$ is a positive integer, $0<\rho<1 \leqq p$, and the series $\Sigma n^{\rho} a_{n}$ is bounded $(C, p)$, that is,

$$
S_{n, p}^{(p)}=r_{n} n^{p}, \quad r_{n}=O(1),
$$

then $\Sigma a_{n}$ satisfies (1.16), where

$$
s=a_{0}+\sum_{\nu=1}^{\infty} r_{\nu} \nu^{p} \Delta^{p+1} \nu^{-p} .
$$

Theorem 14. If $p$ is a positive integer, $0<\rho<1 \leqq p$, and the series $\sum n^{\rho} a_{n}$ is summable $(C, p)$, that is,

$$
S_{n, \rho}^{(p)}=\sigma A_{n}^{(p)}+\epsilon_{n} n^{p}, \quad \epsilon_{n} \rightarrow 0,
$$

then $\Sigma a_{n}$ satisfies (1.17), where $\lambda=0$ and

$$
s=a_{0}-\sigma \sum_{\nu=1}^{p+1} \nu^{-\rho} A_{\nu}^{(-p-2)}+\sum_{\nu=1}^{\infty} \epsilon_{\nu} \nu^{p} \Delta^{p+1} \nu^{-\rho} .
$$


We shall prove only Theorem 14 since the proof of Theorem 13 is similar.

We have

$$
\begin{aligned}
S_{n}^{(p)}= & a_{0} A_{n}^{(p)}+\sum_{\nu=1}^{n} A_{n-\nu}^{(p)} \nu^{-\rho} \nu^{\rho} a_{\nu} \\
= & a_{0} A_{n}^{(p)}+\sum_{\nu=1}^{n} A_{n-\nu}^{(p)} \nu^{-\rho} \sum_{\mu=1}^{\nu} A_{\nu-\mu}^{(-p-2)} S_{\mu, \rho}^{(p)} \\
= & a_{0} A_{n}^{(p)}+\sigma \sum_{\nu=1}^{n} A_{n-\nu}^{(p)} \nu^{-\rho}\left\{\sum_{\mu=0}^{\nu} A_{\nu-\mu}^{(-p-2)} A_{\mu}^{(p)}-A_{\nu}^{(-p-2)}\right\} \\
& \quad+\sum_{\mu=1}^{n} \epsilon_{\mu} \mu^{p} \sum_{\nu=\mu}^{n} A_{n-\nu}^{(p)} \nu^{-\rho} A_{\nu-\mu}^{(-p-2)} \\
= & a_{0} A_{n}^{(p)}+E_{1}+E_{2},
\end{aligned}
$$

say. Clearly

$$
\begin{aligned}
E_{1} & =-\sigma \sum_{\nu=1}^{p+1} A_{n-\nu}^{(p)} \nu^{-\rho} A_{\nu}^{(-p-2)} \\
& =-\sigma A_{n}^{(p)} \sum_{\nu=1}^{p+1} \nu^{-\rho} A_{\nu}^{(-p-2)}+O\left(n^{p-1}\right) \\
& =-\sigma A_{n}^{(p)} \sum_{\nu=1}^{p+1} \nu^{-\rho} A_{\nu}^{(-p-2)}+o\left(n^{p-\rho}\right)
\end{aligned}
$$

Also, by Lemma 5, we have

where

$$
\begin{aligned}
E_{2} & =o\left(n^{p-\rho}\right)+\sum_{\mu=1}^{n-p-1} \epsilon_{\mu} \mu^{p} \sum_{\nu=\mu}^{n} A_{n-\nu}^{(p)} A_{\nu-\mu}^{(-p-2)} \nu^{-\rho} \\
& =o\left(n^{p-\rho}\right)+\sum_{q=0}^{p+1}\left(\begin{array}{c}
p+1 \\
q
\end{array}\right) F_{q}
\end{aligned}
$$

$$
F_{q}=\sum_{\mu=1}^{n-p-1} \epsilon_{\mu} \mu^{p} A_{n-\mu-q}^{(q-1)} \Delta^{q} \mu^{-\rho} .
$$

Now $F_{0}=0$, and, for $q=1,2, \ldots, p$,

$$
F_{q}=o\left\{\sum_{\mu=0}^{n-q} A_{\mu}^{(p-\rho-q)} A_{n-q-\mu}^{(q-1)}\right\}=o\left(n^{p-\rho}\right) .
$$

Also we may write

$$
\begin{aligned}
F_{p+1} & =A_{n-p-1}^{(p)} \sum_{\mu=1}^{\infty} \epsilon_{\mu} \mu^{p} \Delta^{p+1} \mu^{-\rho}-A_{n-p-1}^{(p)} \sum_{\mu=n-p}^{\infty} \epsilon_{\mu}^{\prime} \mu^{p} \Delta^{p+1} \mu^{-\rho} \\
& -\sum_{\mu=1}^{n-p-1}\left\{A_{n-p-1}^{(p)}-A_{n-p-1-\mu}^{(p)}\right\} \epsilon_{\mu} \mu^{p} \Delta^{p+1} \mu^{\prime-p} \\
& =E_{2,1}-E_{2,2}-E_{2,3}
\end{aligned}
$$


say. Now

$$
\begin{aligned}
& E_{2,1}=A_{n}^{(p)} \sum_{\mu=-1}^{\infty} \epsilon_{\mu} \mu^{p} \Delta^{p+1} \mu^{-\rho}+O\left(n^{p-1}\right), \\
& E_{2,2}=o\left\{n^{p} \sum_{\mu=n}^{\infty} \mu^{-p-1}\right\}=o\left(n^{p-\rho}\right),
\end{aligned}
$$

and

$$
E_{2,3}=\sum_{\mu=1}^{n-p-1}\left\{A_{n-p-1}^{(p)}-A_{n-p-1-\mu}^{(p)}\right\}\left\{A \epsilon_{\mu} \mu^{-p-1}+\epsilon_{\mu}^{*} \mu^{-\rho-2}\right\}
$$

where $A$ is a definite non-zero constant and $\epsilon_{n}^{*} \rightarrow 0$. It follows, by Lemma 6, that

$$
E_{2,3}=o\left(n^{p-\rho}\right)+O\left(n^{p-1}\right)=o\left(n^{p-\rho}\right) .
$$

The theorem is therefore proved.

5.4. We now discuss the analogues of Theorems 13 and 14 when $\rho=1$, and we shall see that there is a slight difference between this case and the case $0<\rho<1$.

ThEOREM 15. If $p$ is a positive integer and the series $\sum n a_{n}$ is bounded $(C, p)$, that is,

$$
S_{n, 1}^{(p)}=r_{n} n^{p}, r_{n}=O(1)
$$

then the series $\Sigma a_{n}$ satisfies the relation

$$
S_{n}^{(p)}=s A_{n}^{(p)}+O\left(n^{p-1}\right),
$$

where

$$
s=a_{0}+\sum_{\mu=1}^{\infty} r_{\mu} \mu^{p} \Delta^{p+1} \mu^{-1} .
$$

Theorem 16. If the series $\sum n a_{n}$ is summable $(C, p), p$ a positive integer, to the sum $\sigma$, that is

$$
S_{n, 1}^{(p)}=\sigma A_{n}^{(p)}+\epsilon_{n} n^{p},
$$

then the series $\Sigma a_{n}$ satisfies the relation

$$
S_{n}^{(p)}=s A_{n}^{(p)}+\lambda A_{n}^{(p-1)}+o\left(n^{p-1}\right),
$$

where

$$
\begin{aligned}
& s=a_{0}-\sigma \sum_{\mu=1}^{p+1} A_{\mu}^{(-p-2)} \mu^{-1}+\sum_{\mu=1}^{\infty} \epsilon_{\mu} \mu^{p} \Delta^{p+1} \mu^{-1}, \\
& \lambda=-\sigma .
\end{aligned}
$$

It will be observed that the enunciations of Theorems 15 and 16 are practically the same as those of Theorems 13 and 14 . I have thought it worth while to state the two former theorems in full for 
the purpose of comparison. Moreover the details of the proofs of the two pairs of theorems are essentially different, the case $\rho=1$ containing complications which are absent when $0<\rho<1$. It is sufficient to prove Theorem 16 since the proof of Theorem 15 is similar.

As in previous arguments we have

$$
\begin{aligned}
S_{n}^{(p)}= & a_{0} A_{n}^{(p)}+\sum_{\nu=1}^{n} A_{n-\nu}^{(p)} \nu^{-1} \sum_{\mu=1}^{\nu} A_{\nu-\mu}^{(-p-2)} S_{\mu, 1}^{(p)} \\
= & a_{0} A_{n}^{(p)}+\sum_{\nu=1}^{n} A_{n-\nu}^{(p)} \nu^{-1} \sum_{\mu=0}^{\nu} A_{\nu-\mu}^{(-p-2)} \sigma A_{\mu}^{(p)}-\sigma \sum_{\nu=1}^{n} A_{n-\nu}^{(p)} \nu^{-1} A_{\nu}^{(-p-2)} \\
& \quad+\sum_{\nu=1}^{n} A_{n-\nu}^{(p)} \nu^{-1} \sum_{\mu=1}^{\nu} A_{\nu-\mu}^{(-p-2)} \epsilon_{\mu} \mu^{p} \\
= & a_{0} A_{n}^{(p)}-\sigma \sum_{\nu=1}^{p+1} A_{n-\nu}^{(p)} \nu^{-1} A_{\nu}^{(-p-2)}+\sum_{\mu=1}^{n} \epsilon_{\mu} \mu^{p} \sum_{\nu=\mu}^{n} A_{n-\nu}^{(p)} A_{\nu-\mu}^{(-p-2)} \nu^{-1}
\end{aligned}
$$

Now

whence

$$
A_{n-r}^{(p)}-A_{n}^{(p)}=-\sum_{\nu=n-r+1}^{n} A_{\nu}^{(p-1)}=-r A_{n}^{(p-1)}+o\left(n^{p-1}\right)
$$

$$
\begin{aligned}
S_{n}^{(p)}= & a_{0} A_{n}^{(p)}-\sigma A_{n}^{(p)} \sum_{\nu=1}^{p+1} A_{\nu}^{(-p-2)} \nu^{-1}+\sigma A_{n}^{(p-1)} \sum_{\nu=1}^{p+1} A_{\nu}^{(-p-2)} \\
& +\sum_{\mu=1}^{n} \epsilon_{\mu} \mu^{p} \sum_{\nu=\mu}^{n} A_{n-\nu}^{(p)} A_{\nu-\mu}^{(-p-2)} \nu^{-1}+o\left(n^{p-1}\right) \\
= & \left\{a_{0}-\sigma \sum_{\nu=1}^{p+1} A_{\nu}^{(-p-2)}{ }_{\nu}-1\right\} A_{n}^{(p)}-\sigma A_{n}^{(p-1)}+o\left(n^{p-1}\right)+E_{n},
\end{aligned}
$$

where

where

$$
\begin{aligned}
E_{n} & =\left\{\sum_{\mu=n-p}^{n}+\sum_{\mu=1}^{n-p-1}\right\} \epsilon_{\mu} \mu^{p} \sum_{\nu=\mu}^{n} A_{n-\nu}^{(p)} A_{\nu-\mu}^{(-p-2)} \nu-1 \\
& =o\left(n^{p-1}\right)+\sum_{q=0}^{p+1}\left(\begin{array}{c}
p+1 \\
q
\end{array}\right) F_{q}
\end{aligned}
$$

$$
F_{q}=\sum_{\mu=1}^{n-p-1} \epsilon_{\mu} \mu^{p} A_{n-\mu-q}^{(q-1)} \Delta^{q} \mu^{-1} .
$$

Now $F_{0}=0$ and, for $1 \leqq q \leqq p-1$,

so that

$$
F_{q}=o\left\{\sum_{\mu=0}^{n-q} A_{n-q-\mu}^{(q-1)} A_{\mu}^{(p-q-1)}\right\}=o\left(n^{p-1}\right),
$$

$$
E_{n}=F_{p+1}+(p+1) F_{p}+o\left(n^{p-1}\right) .
$$

We now write

$$
F_{p+1}=G_{1}-G_{2}-G_{3}
$$


where

Clearly

$$
\begin{aligned}
& G_{1}=A_{n-p-1}^{(p)} \sum_{\mu=1}^{\infty} \epsilon_{\mu} \mu^{p} \Delta^{p+1} \mu^{-1} \\
& G_{2}=A_{n-p-1}^{(p)} \sum_{\mu=n-p}^{\infty} \epsilon_{\mu} \mu^{p} \Delta^{p+1} \mu^{-1} \\
& G_{3}=\sum_{\mu=1}^{n-p-1} \epsilon_{\mu} \mu^{p} \Delta^{p+1} \mu^{-1}\left\{A_{n-p-1}^{(p)}-A_{n-p-1-\mu}^{(p)}\right\}
\end{aligned}
$$

and

$$
G_{1}=A_{n}^{(p)} \sum_{\mu=1}^{\infty} \epsilon_{\mu} \mu^{p} \Delta^{p+1} \mu^{-1}-(p+1) A_{n}^{(p-1)} \sum_{\mu=1}^{\infty} \epsilon_{\mu} \mu^{p} \Delta^{p+1} \mu^{-1}+o\left(n^{p-1}\right)
$$

\section{Thus}

$$
G_{2}=o\left\{n^{p} \sum_{\mu=n}^{\infty} \mu^{-2}\right\}=o\left(n^{p-1}\right)
$$

$$
S_{n}^{(p)}=s A_{n}^{(p)}-\left\{\sigma+(p+1) \sum_{\mu=1}^{\infty} \epsilon_{\mu} \mu^{p} \Delta^{p+1} \mu^{-1}\right\} A_{n}^{(p-1)}+H_{n}+o\left(n^{p-1}\right)
$$

where

$$
\begin{aligned}
& H_{n}=\sum_{\mu=1}^{n-p-1} \epsilon_{\mu} \mu^{p}\left[A_{n-p-\mu}^{(p-1)}(p+1) \Delta^{p} \mu^{-1}-\left\{A_{n-p-1}^{(p)}-A_{n-p-1-\mu}^{(p)}\right\} \Delta^{p+1} \mu^{-1}\right] \\
& =(p+1) ! \sum_{\mu=1}^{n-p-1} \frac{\epsilon_{\mu} \mu^{p}}{\mu(\mu+1) \ldots(\mu+p+1)} \\
& {\left[(\mu+p+1) A_{n-p-\mu}^{(p-1)}-\left\{A_{n-p-1}^{(p)}-A_{n-p-1-\mu}^{(p)}\right\}\right]} \\
& =(p+1) ! \sum_{\mu=1}^{n-p-1} \frac{\epsilon_{\mu} \mu^{p}}{\mu(\mu+1) \ldots(\mu+p+1)} \\
& {\left[\frac{(\mu+p+1)(n-p-\mu+1)(n-p-\mu+2) \ldots(n-1-\mu)}{(p-1) !}\right.} \\
& \left.-\left\{\frac{(n-p)(n-p+1) \ldots(n-1)-(n-p-\mu)(n-p-\mu+1) \ldots(n-1-\mu)}{p !}\right\}\right] \\
& =(p+1) ! \sum_{\mu=1}^{n-p-1} \frac{\epsilon_{\mu} \mu^{p}}{\mu(\mu+1) \ldots(\mu+\dot{p}+1)} \sum_{r+8 \leq p} \beta_{r, s} n^{r} \mu^{s},
\end{aligned}
$$

where $\beta_{r, s}$ is a constant independent of $n$ and $\mu$,

and

$$
\beta_{p, 0}=\beta_{p-1,1}=0 \text {, }
$$

We now write

$$
\beta_{p-1,0}=\frac{p+1}{(p-1) !}
$$

where

$$
H_{n}=H_{n}^{(1)}+H_{n}^{(2)}
$$

$$
\begin{aligned}
& H_{n}^{(1)}=\frac{(p+1)}{(p-1) !} n^{p-1} \sum_{\mu=1}^{n-p-1} \epsilon_{\mu} \mu^{p} \Delta^{p+1} \mu^{-1} \\
& H_{n}^{(2)}=(p+1) ! \sum_{\mu=1}^{n-p-1} \frac{\epsilon_{\mu} \mu^{p}}{\mu(\mu+1) \ldots(\mu+p+1)} \sum_{r, s} \beta_{r, s} n^{r} \mu^{s},
\end{aligned}
$$


the summation in $H_{n}^{(2)}$ extending over all values of $r$ and $s$ for which $0 \leqq r \leqq p-2,0 \leqq s \leqq p, r+s \leqq p$.

As $n \rightarrow \infty$ we clearly have

and if we write

$$
H_{n}^{(1)}=A_{n}^{(p-1)}(p+1) \sum_{\mu=1}^{\infty} \epsilon_{\mu} \mu^{p} \Delta^{p+1} \mu^{-1}+o\left(n^{p-1}\right)
$$

$$
H_{n}^{(2)}=H_{n}^{(2,1)}+H_{n}^{(2,2)}+H_{n}^{(2,3)},
$$

where $H_{n}^{(2,1)}$ contains all terms for which $s=0,0 \leqq r \leqq p-2$, $H_{n}^{(2,2)}$ those for which $s=1,0 \leqq r \leqq p-2$ and $H_{n}^{(2,3)}$ those for which $2 \leqq s \leqq p, 0 \leqq r \leqq p-2, r+s \leqq p$, then

and

$$
\begin{aligned}
& H_{n}^{(2,1)}=o\left\{\sum_{r} n^{r} \sum_{\mu=1}^{n} \mu^{-2}\right\}=\sum_{r} o\left(n^{r}\right)=o\left(n^{p-2}\right), \\
& H_{n}^{(2,2)}=o\left\{\sum_{r} n^{r} \sum_{\mu=1}^{n} \mu^{-1}\right\}=\underset{r}{\sum} o\left(n^{r} \log n\right)=o\left(n^{p-1}\right),
\end{aligned}
$$

$$
H_{n}^{(2,3)}=o\left\{\sum_{r, 8} n^{r} \sum_{\mu=1}^{n} \mu^{s-2}\right\}=\sum_{r, 8} o\left(n^{r+s-1}\right)=o\left(n^{p-1}\right) .
$$

The theorem is therefore proved.

5.5. We conclude this section by considering briefly the truth or falsity of the following theorem.

THEOREM 17. A necessury and sufficient condition for the series $\Sigma a_{n}$ to satisfy a relation of the form (1.17), where $p$ is a positive integer and $0<\rho \leqq 1$, is that the series $\Sigma n^{\rho} a_{n}$ should be summable $(C, p)$.

That this theorem is true for the case $\rho=1$ follows at once from Theorems 6 and 16. When $0<\rho<1$ we see, from Theorem 14, that the sufficiency part of Theorem 17 is true and also that the $\lambda$ of relation (1.17) is zero. When $\lambda \neq 0$, Theorem 12 shows that the necessity part of Theorem 17 is false. It is natural therefore to examine the following assertion.

If $0<\rho<1, p$ is a positive integer and the series $\Sigma a_{n}$ satisfies the relation

$$
S_{n}^{(p)}=s A_{n}^{(p)}+\eta_{n}, \eta_{n}=o\left(n^{p-\rho)},\right.
$$

then the series $\Sigma n^{p} a_{n}$ is summable $(C, p)$.

An examination of the proof of Theorem 9 shows that the truth or falsity of this assertion depends, in the last resort, on whether or not the expression

$$
\frac{1}{A_{n}^{(p)}} \sum_{\mu=0}^{n} A_{n-\mu}^{(p)} \eta_{\mu} \Delta^{p+1} \mu^{\rho}
$$

tends to a definite limit; that is, it depends on whether or not the 
series $\Sigma \eta_{\mu} \Delta^{p+1} \mu^{\rho}$ is summable $(C, p)$. It is clear that this series need not be summable $(C, p)$. Indeed, if

$$
\eta_{\mu}=\frac{\mu^{p-\rho}}{\log (\mu+1)},
$$

it is properly divergent. Hence the necessity part of Theorem 17 is not true for $0<\rho<1$ even when $\lambda=0$. In other words, either of the alternatives in the conclusion of Theorem 11 may occur.

6.1. It is natural to expect that series which satisfy (1.16) and (1.17) should have a less restrictive Tauberian condition than $a_{n}=O\left(n^{-1}\right)$. It was proved ${ }^{1}$ by Hardy that series, for which

$$
S_{n}^{(1)}=s A_{n}^{(1)}+o\left(n^{\frac{1}{4}}\right),
$$

are summable by Borel's method. Hence a sufficient Tauberian condition for such series is

$$
a_{n}=O\left(n^{-\frac{1}{2}}\right) .
$$

More generally ${ }^{2}$, it has been proved $^{3}$ recently that, if the series $\Sigma a_{n}$ satisfies (1.17) with $\lambda=0$, it is summable by Valiron's method ${ }^{4}$ of order $1-\rho / p$, where $0<\rho<p$. An examination of the argument used in the proof of this theorem shows at once that the theorem remains true when $\Sigma a_{n}$ satisfies (1.17) with $\lambda \neq 0$. Now the Tauberian condition for summability $(V, a)$, where $0<a<1$, is known ${ }^{5}$ to be

$$
a_{n}=O\left(n^{-a}\right) .
$$

Hence we have the theorem.

Theorem 18. If $\Sigma a_{n}$ satisfies (1.17), where $p$ is a positive integer, and $0<\rho<p, 0<\rho \leqq 1$, and if

$$
a_{n}=O\left\{n^{(p-p) ! p}\right\},
$$

the series $\Sigma a_{n}$ is convergent.

This theorem may be proved directly by making use of an argument due to Hardy and Littlewood ${ }^{6}$, and the same type of

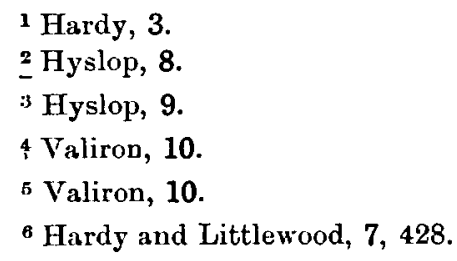


argument may also be used to prove an analogous theorem for series satisfying (1.16).

ThEOREM 19. If $\Sigma a_{n}$ satisfies (1.16), where $p$ is a positive integer and $0<\rho<p, 0<\rho \leqq 1$, and if

$$
a_{n}=o\left\{n^{(\rho-p) / p}\right\},
$$

the series $\Sigma a_{n}$ is convergent.

\section{REFERENCES.}

1. A. F. Andersen, Studier over Cesàro's Summabilitetsmetode (Copenhagen, 1921).

2. H. Bohr, Bidrag til de Dirichlet'ske Raekkers Theori (Copenhagen, 1910).

3. G. H. Hardy, Researches in the Theory of Divergent Series and Divergent Integrals, Quart. $J$. of Math., 35 (1904), 22-66.

4. G. H. Hardy, On certain Oscillating Series, Quart. J. of Math., 38 (1907), 269.288.

5. G. H. Hardy, Generalization of a Theorem in the Theory of Divergent Series, Proc. London Math. Soc. (2), 6 (1908), 255-264, and footnote (2) 8 (1909), 277-294.

6. G. H. Hardy, Theorems relating to the Summability and Convergence of slowly oscillating Series, Proc. London Math. Soc. (2), 8 (1910), 301-320.

7. G. H. Hardy and J. E. Littlewood, Contributions to the Arithmetic Theory of Series, Proc. London Math. Soc. (2), 11 (1913), 411-478.

8. J. M. Hyslop, The Generalization of a Theorem on Borel Summability, Proc. London Math. Soc. (2), 41 (1936), 243-2ä6.

9. J. M. Hyslop, On the Summability of Series by a Method of Valiron, Proc. Edinburgh Math. Soc. (2), 4 (1936), 218-223.

10. G. Valiron, Remarques sur la Sommation des Séries Divergentes par les Méthodes de M. Borel, Rendiconti di Palermo, 42 (1917), 267-284.

The University, Glasgow. 